\title{
Tracing TET1 expression in prostate cancer: discovery of malignant cells with a distinct oncogenic signature
}

\author{
U. Schagdarsurengin ${ }^{1,2 \dagger}$, C. Luo ${ }^{1 \dagger}$, H. Slanina ${ }^{3}$, D. Sheridan ${ }^{4}$, S. Füssel ${ }^{5}$, N. Böğürcü-Seidel ${ }^{6}$, S. Gattenloehner ${ }^{4}$, \\ G. B. Baretton ${ }^{7}$, L. C. Hofbauer ${ }^{8}$, F. Wagenlehner ${ }^{1}$ and T. Dansranjav ${ }^{1 *}$ (D)
}

\begin{abstract}
Background: Ten-eleven translocation methylcytosine dioxygenase 1 (TET1) is involved in DNA demethylation and transcriptional regulation, plays a key role in the maintenance of stem cell pluripotency, and is dysregulated in malignant cells. The identification of cancer stem cells (CSCs) driving tumor growth and metastasis is the primary objective of biomarker discovery in aggressive prostate cancer (PCa). In this context, we analyzed TET1 expression in PCa.
\end{abstract}

Methods: A large-scale immunohistochemical analysis of TET1 was performed in normal prostate (NOR) and PCa using conventional slides (50 PCa specimens) and tissue microarrays (669 NOR and 1371 PCa tissue cores from 371 PCa specimens). Western blotting, RT-qPCR, and $450 \mathrm{~K}$ methylation array analyses were performed on PCa cell lines. Genome-wide correlation, gene regulatory network, and functional genomics studies were performed using publicly available data sources and bioinformatics tools.

Results: In NOR, TET1 was exclusively expressed in normal cytokeratin 903 (CK903)-positive basal cells. In PCa, TET1 was frequently detected in alpha-methylacyl-CoA racemase (AMACR)-positive tumor cell clusters and was detectable at all tumor stages and Gleason scores. Pearson's correlation analyses of PCa revealed 626 TET1-coactivated genes $(r>0.5)$ primarily encoding chromatin remodeling and mitotic factors. Moreover, signaling pathways regulating antiviral processes (62 zinc finger, ZNF, antiviral proteins) and the pluripotency of stem cells were activated. A significant proportion of detected genes exhibited TET1-correlated promoter hypomethylation. There were 161 genes encoding transcription factors (TFs), of which 133 were ZNF-TFs with promoter binding sites in TET1 and in the vast majority of TET1-coactivated genes.

Conclusions: TET1-expressing cells are an integral part of PCa and may represent CSCs with oncogenic potential. Keywords: Prostate cancer, TET1, Oncogene, ZNF transcription factor, ZNF antiviral protein

\section{Background}

Ten-eleven translocation (TET) family dioxygenases are the main catalysts responsible for the oxidation of 5-methylcytosine $(5 \mathrm{mC})$ in DNA to

\footnotetext{
${ }^{*}$ Correspondence: Temuujin.Dansranjavin@chiru.med.uni-giessen.de

${ }^{\dagger} U$. Schagdarsurengin and C. Luo have contributed equally to this work

${ }^{1}$ Clinic of Urology, Pediatric Urology and Andrology, Justus-LiebigUniversity Giessen, Giessen, Germany

Full list of author information is available at the end of the article
}

5-hydroxymethylcytosine $(5 \mathrm{hmC})$ and further oxidation products. TET DNA demethylases and their catalytic products are key regulators of embryonic development, stem cell functions, and lineage specification [1]. Safeguarding bivalent promoters from de novo methylation is one of the key functions of TETs in stem cells, and a number of chromatin immunoprecipitation (ChIP) and knock-out experiments in human and mouse embryonic stem cells have demonstrated that in particular, TET1 
and Tet1, respectively, bind to hypomethylated promoters of pluripotency-related genes and maintain their active status [2-5]. The essential role of TET1/Tet1 in maintaining pluripotency and regulating differentiation has been shown in different stem cell types, for example, embryonic [5, 6], neural [7], mesenchymal [8], trophoblast $[9,10]$, and hematopoietic stem cells [11].

In normal prostate epithelium, the stem cell population is located in the proliferative basal cell compartment. The self-renewing basal stem cells give rise to bipotent basal progenitors which are precursor cells of prostatic secretory (luminal) cells [12]. In PCa, cancer stem cells (CSCs) capable of self-renewal and asymmetric division and having adaptive capacity have been postulated to be responsible for tumor growth and metastasis [13-15], tumor survival after chemotherapeutics and radiation, and initiation of therapeutic relapse [16-18]. Despite basic common features attributed to cell stemness, CSCs in PCa exhibit a pronounced molecular heterogeneity displayed in a large number of extracellular and intracellular markers [15]. Thus, in order to develop therapeutic strategies for $\mathrm{PCa}$, there is still great interest in deeper understanding of molecular mechanisms responsible for establishment and maintenance of CSCs.

The balance between $5 \mathrm{mC}$ and unmethylated cytosine is needed in order to maintain stem cell self-renewal as well as upon cell differentiation $[2,19,20]$. In human malignancies, TET1 has been identified as a fusion partner of the mixed-lineage leukemia gene in acute myeloid leukemia [21]. Dysregulation of TET1 expression or function and aberrant methylation have been observed in a wide range of cancers, for example, gastric cancer, thyroid carcinoma, lung cancer, gliomas, esophageal carcinoma, and breast cancer [22-27]. With regard to PCa, data are still scarce. In PCa tissues, TET1 has been found to be downregulated, and in xenograft models, TET1 depletion facilitates tumor growth and $\mathrm{PCa}$ metastasis [28]. Furthermore, in high-risk PCa, TET1 mutation and mRNA downregulation are associated with worse metastasis-free survival [29]. However, existing studies on TET1 comprise a relatively low number of primary $\mathrm{PCa}$ specimens, and the role of TET1-expressing cells in $\mathrm{PCa}$ remains unclear.

Here, we describe a large-scale TET1 protein expression study in normal prostate (NOR) and $\mathrm{PCa}$, and a genome-wide correlation and regulatory network study using different publicly accessible data sources and bioinformatics tools. Our data demonstrate striking differences in the TET1 expression profiles of NOR and PCa, clarify the epigenetic background of TET1-upregulation in PCa, identify all TET1-coactivated genes and demethylated promoters, and provide their functional characterization. All in all, our data suggest that TET1-expressing cell clusters in PCa represent CSCs with distinct oncogenic potential.

\section{Methods}

Immunohistochemical analysis of TET1 expression

Conventional slides from formalin-fixed, paraffinembedded tissue blocks were prepared from specimens obtained from 50 PCa patients who underwent radical prostatectomy at the Department of Urology, Pediatric Urology and Andrology (Justus-Liebig-University Giessen, Germany). All patients gave their written consent, and the study was approved by the ethics committee of the Medical Faculty (Justus-Liebig-University Giessen, Germany) (Ethics Vote: 49/05). All tissue samples were examined and characterized at the Institute of Pathology (Justus-Liebig-University Giessen, Germany). The PCa specimens were characterized with regard to the pathologic stage, differentiation grade (Gleason score), presence of lymph node metastases, and residual tumor (Table 1). Tissue microarrays (TMA) originating from formalin-fixed, paraffin-embedded tissue blocks of 371 radical prostatectomy specimens were obtained from the Department of Urology of Technical University Dresden (Table 2). For each PCa patient, the TMA included four tissue cores from cancerous areas (PCa-TMA) and two tissue cores from non-cancerous areas (NOR-TMA).

For immunohistochemical studies (IHC), slides of paraffin-embedded tissue were deparaffinized using xylene and then rehydrated by successive incubation in $100 \%, 96 \%$, and $70 \%$ ethyl alcohol and then $\mathrm{ddH}_{2} \mathrm{O}$. Next, slides were immersed in $1 \%$ hydrogen peroxide $\left(\mathrm{H}_{2} \mathrm{O}_{2}\right)$ for $20 \mathrm{~min}$ to block endogenous peroxidase activity. Sections were washed once in $1 \mathrm{X}$ Tris- $\mathrm{HCl}$ buffer. For antigen retrieval, sections were incubated in citrate buffer (pH 6.0) and heated in a water bath at $70{ }^{\circ} \mathrm{C}$ for $15 \mathrm{~min}$. Subsequently, sections were incubated overnight at $4{ }^{\circ} \mathrm{C}$

Table 1 Clinical data of 50 PCa patients analyzed by IHC using conventional paraffin slides with regard to TET1 expression $(n=50$ patients in total)

\begin{tabular}{lclllll}
\hline & Count (\%) & \multicolumn{2}{l}{ Age $(\mathbf{y})$} & & \multicolumn{2}{c}{ PSA $(\mathbf{n g} / \mathbf{m L})$} \\
\cline { 7 - 7 } & & Median & Range & & Median & Range \\
\hline T2a-C & $26(52.0 \%)$ & 64.24 & $47.8-75.1$ & & 7.0 & $0.1-54.8$ \\
T3a-b & $24(48.0 \%)$ & 64.16 & $55.5-74.9$ & & 9.0 & $0.6-27.2$ \\
GS6 & $23(46.0 \%)$ & 65.88 & $47.8-75.1$ & & 8.8 & $0.1-24.8$ \\
GS7 & $20(40.0 \%)$ & 63.93 & $54.8-73.1$ & & 8.8 & $0.8-54.8$ \\
GS8 & $2(4.0 \%)$ & 65.53 & $58.2-72.8$ & 6.0 & $2.7-9.3$ \\
GS9 & $5(10.0 \%)$ & 65.20 & $55.5-74.9$ & & 14.1 & $6-22.1$ \\
\hline
\end{tabular}

PCa prostate cancer, ICH immunohistochemical analysis, TET1 ten-eleven translocation family member $1, y$ years, $P S A$ prostate-specific antigen, $T$ tumor stage, GS Gleason score 
Table 2 Clinical data of PCa patients analyzed by IHC using TMA with regard to TET1 expression ( $n=371$ patients in total)

\begin{tabular}{|c|c|c|c|c|c|}
\hline & \multirow[t]{2}{*}{ Count (\%) } & \multicolumn{2}{|l|}{ Age (y) } & \multicolumn{2}{|c|}{ PSA (ng/mL) } \\
\hline & & Median & Range & Median & Range \\
\hline Low risk & 97 (26.2\%) & 64.6 & $48.5-78.3$ & 5.74 & $0.2-38.4$ \\
\hline Intermed. risk & 45 (12.1\%) & 64.9 & $50.6-76.9$ & 12.9 & $2.9-83.8$ \\
\hline High risk & $229(61.7 \%)$ & 65.5 & $46.7-77.4$ & 9.9 & $0.3-113$ \\
\hline T2a-c & $165(44.5 \%)$ & 65.1 & $46.7-78.3$ & 7.2 & $0.2-45.1$ \\
\hline T3a-b & $144(38.8 \%)$ & 65.9 & $54.1-77.4$ & 8.7 & $0.3-74.8$ \\
\hline T4 & $62(16.7 \%)$ & 63.2 & $50.6-76.5$ & 15.8 & $2.8-113$ \\
\hline$G S \leq 6$ & 73 (19.7\%) & 64.3 & $48.5-77.1$ & 5.6 & $0.2-83.8$ \\
\hline GS7 & $69(18.6 \%)$ & 65.6 & $50.6-78.3$ & 8.0 & $2.9-50$ \\
\hline GS8 & $147(39.6 \%)$ & 66.1 & $46.7-77.4$ & 10.5 & $0.3-113$ \\
\hline $\mathrm{GS} \geq 9$ & $82(22.1 \%)$ & 64.6 & $51.1-77.1$ & 8.8 & $0.3-65.5$ \\
\hline
\end{tabular}

$P C a$ prostate cancer, IHC immunohistochemical analysis, TMA tissue microarray, TET1 ten-eleven translocation family member 1, y years, PSA prostate-specific antigen, $T$ tumor stage, GS Gleason score, Intermed. risk intermediate risk

with the primary antibodies diluted in $0.1 \%$ non-fat milk in $1 \mathrm{X}$ Tris- $\mathrm{HCl}$ buffer. We characterized prostate cells on conventional slides using antibodies to TET1 (anti-TET1 rabbit polyclonal antibody, 1:400, GeneTex, GTX124207), the prostate basal cell marker cytokeratin 903 (CK903) (anti-34betaE12, $5 \mu \mathrm{g} / \mathrm{mL}$, Leica Biosystems, Germany) and the prostate cancer cell marker alpha-methylacylCoA racemase (AMACR) (anti-AMACR, $10 \mu \mathrm{g} / \mathrm{mL}$, Dako, Germany). After washing with $2 \%$ Triton/PBS buffer, the slides were incubated with biotinylated secondary antibodies (anti-rabbit IgG antibody, 1:500; antimouse IgG antibody, 1:1000; Dako, Germany) for $1 \mathrm{~h}$ at room temperature and washed three times with PBS buffer for $5 \mathrm{~min}$. Next, slides were incubated for $30 \mathrm{~min}$ with an avidin-biotin complex system (Vector Laboratories, Burlingame, CA, USA), and bound antibody was detected using the 3-amino-9-ethylcarbazole substrate. Conventional slides were analyzed using an Olympus BX43 light microscope, and TMA slides were analyzed using ZEN 2.3 (blue edition) software after scanning with a Zeiss Axio Scan.Z1 slide scanner (Zeiss Microscopy).

\section{Cell culture}

Human PCa cell lines PC3, LNCaP, and DU145 were obtained from the German Resource Center for Biological Material (DSMZ, Braunschweig, Germany). DU145 and LNCaP were cultured in RPMI 1640 medium (Gibco), and PC3 in DMEM (Gibco) supplemented with $10 \%$ fetal bovine serum (Gibco) and 1\% penicillin/streptomycin (Gibco). Cells were cultured in 10-cm dishes in $5 \% \mathrm{CO}_{2}$ at $37{ }^{\circ} \mathrm{C}$ to $90-100 \%$ confluency, then used for western blot, mRNA expression, and genome-wide methylation analyses.

\section{Analysis of TET1 expression in PCa cell lines by western} blot

For protein extraction, PC3, DU145, and LNCaP cells were washed with $\mathrm{PBS}$ buffer and lysed in lysis buffer (10 mM Tris- $\mathrm{HCl}, 150 \mathrm{mM} \mathrm{NaCl}, 1 \mathrm{mM}$ EDTA, $1 \mathrm{mM}$ DTT, $1 \mathrm{mM}$ phenylmethylsulfonylfluoride, and $1 \%$ Triton X-100, pH 7.4) supplemented with complete protease inhibitor cocktail tablets (Roche). After an incubation on ice for $30 \mathrm{~min}$, cell debris was removed by centrifugation at $12,000 \mathrm{~g}$ for $20 \mathrm{~min}$, and protein was quantified using the Pierce BCA protein assay (Thermo Fisher). In total, $10 \mu \mathrm{g}$ of protein was mixed with $5 \mu \mathrm{L}$ loading buffer $(4.5 \mu \mathrm{L}$ Laemmli buffer and $0.5 \mu \mathrm{L} \beta$-mercaptoethanol), heated at $95{ }^{\circ} \mathrm{C}$ for $5 \mathrm{~min}$, and separated in a polyacrylamide gel (resolving gel 7.5\%, stacking gel 4\%). Semi-dry transfer (Trans-Blot SD Semi-dry transfer cell, Bio-Rad) was performed for $1 \mathrm{~h}$ at $150 \mathrm{~mA}(\max .25 \mathrm{~V}$ ) onto polyvinylidenedifluoride membranes (Merck-Millipore). Blocking was performed using Odyssey blocking buffer (1:3 diluted in PBS) for $1 \mathrm{~h}$ at room temperature. The membranes were incubated overnight at $4{ }^{\circ} \mathrm{C}$ with TET1 $(1: 2000$, GeneTex, GTX124207) and GAPDH antibodies (1:2500, Abcam, ab9485) diluted in Odyssey blocking buffer (1:3 diluted in PBS, plus 0.1\% Tween-20). Membranes were washed in PBS-Tween-20 buffer $(0.1 \%$ Tween-20 in $\mathrm{PBS}$ ) and incubated with IRDye-conjugated secondary antibodies (LI-COR Biosciences) for $1 \mathrm{~h}$ at room temperature. After washing in PBS-Tween-20 buffer, the membranes were rinsed with PBS and the fluorescence signals were detected using the Odyssey Fc imaging system.

\section{Analysis of TET1 expression in PCa cell lines by RT-qPCR}

The total RNA was extracted from PC3, DU145, and LNCaP cells using the peqGOLD TriFast reagent (VWR) according to manufacturer's instructions. Reverse transcription was performed on $1 \mu \mathrm{g}$ total RNA using M-MLV transcriptase and adjusted buffer system (Promega), random hexamers, and poly-dT primers for $1 \mathrm{~h}$ at $42{ }^{\circ} \mathrm{C}$. The resulting cDNAs were purified using a QIAquick PCR purification kit (Qiagen), and the concentrations were measured. Real-time PCRs were performed using $50 \mathrm{ng}$ of cDNA per PCR in a Rotor-Gene Q PCR Cycler (Qiagen) for TET1 (forward primer: $5^{\prime}$-TCCTGGTGCTAT TCCAGTCC- 3 ', reverse primer: 5'-CAGGAAGGAAGA CAGGCAAG- $3^{\prime}$, product size: 110 base pairs) with the reference gene GAPDH (forward primer: 5'-TGGAGA AGGCTGGGGCTCAT-3', reverse primer: $5^{\prime}$-GACCTT GGCCAGGGGTGCTA-3', product size: 176 base pairs). TET1 expression was calculated as a relative expression by normalization to GAPDH using the $2^{-\triangle \Delta \mathrm{Ct}}$ method. 


\section{Genome-wide methylation analyses in PCa cell lines}

Genome-wide methylation analysis of PC3, DU145, and LNCaP cells was performed using the Illumina Infinium HumanMethylation $450 \mathrm{~K}$ BeadChip platform provided by Life \& Brain GmbH. The DNA was extracted and purified from PCa cell lines using a DNA Mini kit (Qiagen), and $1 \mu \mathrm{g}$ of DNA was used for each methylation analysis. Import of raw Illumina $450 \mathrm{~K}$ BeadChip IDAT files, data preprocessing, and correction and normalization steps were carried out using the $\mathrm{R}$ computing environment (v. 4.0) with the Bioconductor package "minf" (v. 1.36.0). The methylation score of each CpG was represented as a beta $(\beta)$-value.

\section{Analysis of viral infections in PCa and BPH tissues}

Tissue samples from $\mathrm{PCa}$ and $\mathrm{BPH}$ were tested for the human cytomegalovirus (HCMV), Epstein-Barr virus (EBV), herpes simplex viruses 1 and 2 (HSV1/2), and JC and BK viruses. The DNA of HCMV and EBV was positively tested and further analyzed in $34 \mathrm{PCa}$ and $16 \mathrm{BPH}$ specimens. Approximately, 10 to $25 \mu \mathrm{g}$ DNA was used as a template for the artus CMV-LC-PCR and artus EBV-LC-PCR kit (Qiagen), respectively. PCRs were performed according to the manufacturer's instructions and calibrated against the WHO first International Standard for Human Cytomegalovirus (NIBSC, code 09/162), and the WHO first International Standard for Epstein-Barr Virus (NIBSC, code 09/260), detecting a quantifiable minimum of 80 genomes $/ \mathrm{mL}$ for $\mathrm{HCMV}$ and 100 genomes/mL for $\mathrm{EBV}$. Positive PCR results below these thresholds were therefore only reported as $<80$ or $<100$ positive. An internal control excluded inhibitory compounds in the sample.

\section{Data sources, application, and bioinformatic evaluation}

To explore the molecular background of $\mathrm{PCa}$ specimens exhibiting high TET1 expression, we employed the Cancer Genome Atlas (TCGA) database (tcgaportal.org), in particular the Illumina Infinium HumanMethylation $450 \mathrm{~K}$ BeadChip data and genome-wide RNA-sequencing data of $341 \mathrm{PCa}$ (adenocarcinoma) and 35 NOR. Supplemental clinical data, raw methylation data ("IDAT" files), and RNA-seq data ("HTSeqcounts" files) were extracted from TCGA using the $R$ package "TCGAbiolinks" (v. 2.18.0). Data preprocessing and subsequent analyses were performed using the $R$ packages "minfi" (v. 1.36.0) for Illumina $450 \mathrm{~K}$ data and "Biobase" (v.2.50) for RNA sequence data. Differential expression and differential methylation analyses were performed using the eBayes function in the "limma" package. $p$ values were adjusted for multiple comparisons using the Benjamini-Hochberg method.

Methylation $(\beta)$ values of all TET1 CpG probes in TCGA (in total 30) covering the promoter, $5^{\prime}$-untranslated region (5'-UTR), and gene body of TET1 were correlated to TET1 expression using data from $341 \mathrm{PCa}$ and 35 NOR, using Spearman's correlation. All differentially methylated TET1 CpG probes in PCa versus NOR were evaluated (Mann-Whitney $U$ test). The PCa specimens from TCGA were divided according to TET1 expression and promoter methylation levels into three groups: "TET1-high" (expression $>$ 85th percentile, hypomethylated, $n=51$ ), "TET1-low" (expression $<40$ th percentile, hypermethylated, $n=136)$ and "TET1-moderate" (intermediate expression and methylation). All differentially methylated TET1 CpG probes in TET1-high versus TET1-low PCa were evaluated (Mann-Whitney U test). Using $341 \mathrm{PCa}$, TET1 expression was tested for correlation with the expression of all protein-encoding genes (Pearson's correlation), and genes showing strong positive correlation (referred as TET1-coactivated genes) were further analyzed with regard to promoter methylation in TET1-high versus TET1-low PCa (Mann-Whitney $U$ test). The statistical analyses are described in detail below.

The Human Transcription Factors databank (http:// humantfs.ccbr.utoronto.ca/index.php) [30] was used to identify TET1-coactivated genes encoding transcription factors (TFs). JASPAR 2020, a database of curated non-redundant TF-binding profiles stored as position frequency matrices (jaspar2020.genereg.net), was used to analyze the binding scores and binding motifs of TFs. Promoter sequences (the 1500 base pairs upstream from the transcription start site, TSS1500) of TET1 and TET1-coactivated genes were extracted and scanned for binding sites for TET1-coactivated TFs. The significance ( $p$ values) of TF-binding sites (TF-BSs) was calculated using the R package "TFMPvalue" (v. 0.0.8) based on the method described by Touzet and Varre [31], and statistically significant TF-BSs $(p<0.01)$ were considered true. Transcription regulatory modules (TRMs) were identified using the R package "rTRM" (v. 1.28.0) [32].

Functional genomics studies were performed on TET1coactivated genes in PCa. Gene ontology (GO) analysis was done using the Database for Annotation, Visualization and Integrated Discovery (DAVID) [33, 34]. Gene set enrichment analysis (GSEA) was done using the Molecular Signatures Database (MSigDB) [35]. Pathway analysis was done using the Kyoto Encyclopedia of Genes and Genomes (KEGG) [36]. Protein-protein interaction (PPI) and PPI network (interactome) analyses were done on TET1-coactivated TFs using the BioGRID database of protein, genetic, and chemical interactions [37]. 
In order to assess whether TET1-coactivated genes in PCa may also possess TET1-binding sites in their promoters, we selected Tet1 ChIP-seq data generated on mouse trophoblast stem cells (GSE109545) [9]. Genes having Tet1-binding sites in their TSS1500 promoters were considered for further analysis.

\section{Statistical analyses}

The R function "rcorr" from the "Hmisc" package was used for correlation analyses. Correlation coefficients between TET1 expression and the expression of 19,252 protein-coding genes were calculated for 341 PCa using Pearson's method, and genes showing a strong positive correlation $(r>0.5, p<0.001)$ were considered to be TET1-coactivated genes. Correlation between gene expression and promoter methylation was calculated using Spearman's method. The Mann-Whitney U test was used for between-group comparisons (e.g., PCa versus NOR, TET1-high versus TET1-low PCa) of TET1 methylation at $30 \mathrm{CpG}$ sites. The Kruskal-Wallis test was used to compare TET1 expression and TET1 methylation at $30 \mathrm{CpG}$ sites among different PCa stages (T2a-c, T3a-b, and T4) and Gleason grades (GS6, GS7, GS8, and $G S \geq 9)$. Statistical significance was adjusted using the Bonferroni method, and $p$ values $<0.05$ were considered significant.

\section{Results}

\section{TET1-expressing cells and cell clusters are frequently} encountered in $\mathrm{PCa}$ and seldom present in benign prostate tissues

Using conventional tissue slides, we examined TET1 expression by IHC in NOR and PCa specimens obtained from 50 PCa patients (Table 1, Fig. 1A). Particular regard was given to the cell specificity of TET1 expression. The basal cell marker CK903 and PCa cell marker AMACR were examined in parallel to TET1. In NOR, TET1 was exclusively expressed in CK903-positive basal cells, and TET1-expressing cells were scattered in the basal epithelium (Fig. 1A.1 and Additional file 1: Fig. S1A). In contrast, in PCa, TET1 was abundantly expressed exclusively in AMACR-positive cancer cells, whereby not all AMACR-positive cells expressed TET1 (Fig. 1A.3 and Additional file 1: Fig. S1C).

For a comprehensive overview, we performed a largescale IHC study of TET1 expression using TMAs representing NOR and PCa specimens from 371 PCa patients (Table 2). In total, we could analyze 1371 single PCaTMA spots and 669 single NOR-TMA spots. Observations in TMAs confirmed the results obtained from IHC analyses using conventional slides. In NOR-TMA, TET1 showed a scattered expression pattern in basal epithelial cells (Fig. 1A.2 and Additional file 1: Fig. S1B). In
PCa-TMA, TET1-expressing cells appeared much more frequently and formed clusters (Fig. 1B, C.1, and Additional file 1: Fig. S1D). In both NOR and PCa, TET1 expression was detectable in the cytoplasm (Fig. 1B.1) as well as in the nucleus (Fig. 1B.2). Single PCa- and NORTMA spots could be categorized with respect to numbers of TET1-expressing cells as "TET1-high" (presence of numerous TET1-expressing cell clusters) (Fig. 1C.1), "TET1-moderate" (presence of a few scattered TET1expressing cells) (Fig. 1C.2), or "TET1-negative" (absence of TET1-expressing cells) (Fig. 1C.3). From $371 \mathrm{PCa}$ patients, 65 (17.5\%) exhibited at least one PCa-TMA spot with high TET1 expression, 160 (43.1\%) exhibited at least one PCa-TMA spot with moderate TET1 expression (Fig. 1C.1, C.2), and 146 (39.4\%) exhibited only TET1negative PCa-TMA spots (Fig. 1C.3). Among 366 PCa patients for which NOR-TMA were analyzed, ten (3\%) exhibited at least one NOR-TMA spot with high TET1 expression, 181 (50\%) at least one NOR-TMA spot with moderate TET1 expression, and 175 (48\%) exhibited only TET1-negative NOR-TMA spots (Fig. 1C.4). Comparison of $\mathrm{PCa}$ patients considering the tumor stages (pT2 to pT4) and Gleason scores (GS $\leq 6$ to $\geq 9)$ revealed that "TET1-high" foci were detectable in $\mathrm{PCa}$ at any tumor stage and Gleason grade (Fig. 1C.4). The frequencies of occurrence of TET1-high, moderate, and negative TMA spots were similar in PCa specimens from tumors of different stages and Gleason grades (Fig. 1C.4).

\section{Upregulation of TET1 in PCa is caused by aberrant DNA methylation in the TET1 promoter, 5'-UTR, and gene body}

Cancer-associated up- and downregulation of proteins is often caused by epigenetic dysregulation of the genes. To understand the epigenetic reasons for TET1 upregulation in PCa, we used TCGA, in particular the methylome and transcriptome data generated on $341 \mathrm{PCa}$ and $35 \mathrm{NOR}$ specimens (Additional file 2: Table S1).

An initial comparison of TET1 expression in $\mathrm{PCa}$ and NOR without any categorization of tissue samples showed no significant differences (Additional file 1: Fig. S2A). A comparison of $\mathrm{PCa}$ samples with regard to TET1 expression in consideration of Gleason grade and tumor stage also showed no significant differences (Additional file 1: Fig. S2B). As methylation changes leading to aberrant gene expression may happen at specific sites throughout a gene, we aimed to systematically analyze all available TET1 CpG probes in TCGA and to identify those critically important for TET1 expression. Altogether, $30 \mathrm{CpG}$ probes, including 8 in the promoter (TSS200 and TSS1500), 16 in the 5'-UTR, and 6 in the gene body of TET1 were available in TCGA (Additional file 2: Table S2). Spearman's analysis of possible correlation between TET1 methylation at $30 \mathrm{CpG}$ probes and 


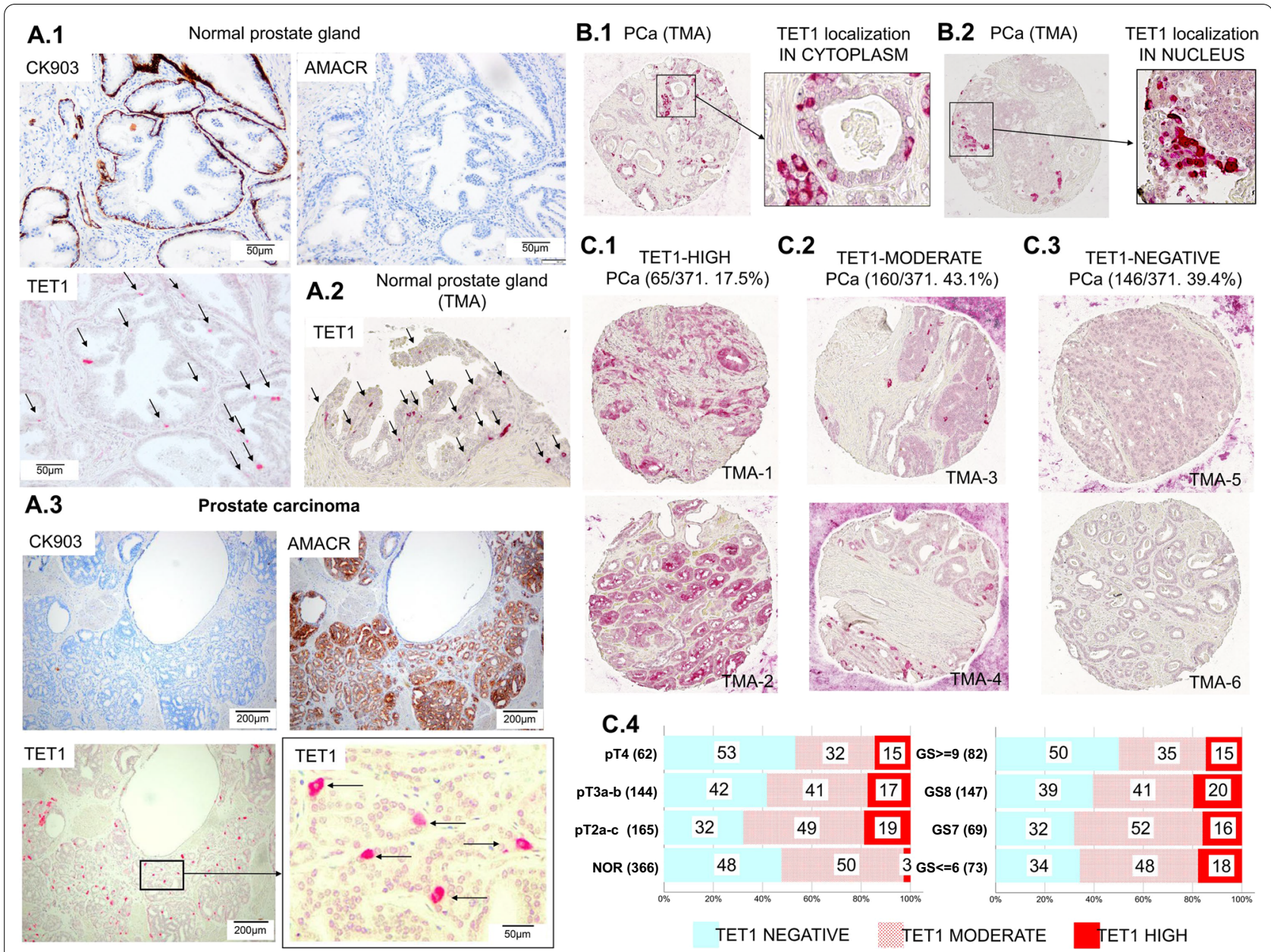

Fig. 1 Analysis of TET1 expression by immunohistochemistry using conventional paraffin slides and tissue microarrays (TMA) in normal prostate (NOR) and prostate carcinoma tissue (PCa). A In NOR, TET1 was expressed in CK903-positive and AMACR-negative normal basal epithelial cells; these TET1-positive cells were rare and scattered in the basal epithelium (A.1 and A.2). In PCa, TET1 was expressed in CK903-negative and AMACR-positive cancer cells, and TET1-expressing cells appeared much more frequently than in NOR (A.3); B TET1 expression was detected in both the cytoplasm (B.1) and the nucleus (B.2); C single NOR- and PCa-TMA spots were categorized according to the TET1 expression level as TET1-high (C.1, e.g., TMA-1 and TMA-2), TET1-moderate (C.2, e.g., TMA-3 and TMA-4), or TET1-negative (C.3, e.g., TMA-5 and TMA-6). The frequencies of TET1-high, moderate, and low TMA spots were calculated among NOR in comparison with PCa in regard to tumor stage (T2 to T4) and Gleason score (GS6 to GS9) (C.4)

TET1 expression revealed the methylation at $4 \mathrm{CpG}$ sites in NOR (one in the promoter and 3 in the $5^{\prime}$-UTR) and $10 \mathrm{CpG}$ sites in $\mathrm{PCa}$ (4 in the promoter, 3 in the $5^{\prime}$-UTR, and 3 in the gene body) to be significantly correlated with TET1 expression (Fig. 2A.1 and Additional file 2: Table S3). In NOR, methylation of all four CpG sites was negatively correlated with TET1 expression (i.e., the more they were methylated, the less the gene was expressed). In contrast, in PCa, methylation of 6 out of 10 detected CpG sites was negatively correlated with TET1 expression, and methylation of 4 was positively correlated (i.e., the more methylation, the more TET1 was expressed) (Fig. 2A.1 and Additional file 2: Table S3). These data show the complexity of TET1 dysregulation in PCa. Further differential methylation analyses of 30 TET1 CpG probes in PCa versus NOR revealed 18 significantly hypermethylated $\mathrm{CpG}$ probes in $\mathrm{PCa}$ (4 in the promoter, 12 in the $5^{\prime}-$ UTR, and 2 in the gene body) and 4 significantly hypomethylated $\mathrm{CpG}$ sites in $\mathrm{PCa}$ (one in the $5^{\prime}$-UTR and 3 in the gene body) (Fig. 2A.1, Additional file 2: Table S3 and Additional file 1: Fig. S3). Comparison of PCa specimens with regard to Gleason scores revealed 7 CpG sites in TET1 ( 2 in the promoter and 5 in the gene body) to be significantly hypermethylated or hypomethylated in the course of prostate carcinogenesis (Additional file 1: Fig. S3).

Next, we selected the TET1 CpG probe showing the most significant correlation with TET1 expression in $\mathrm{PCa}$ 

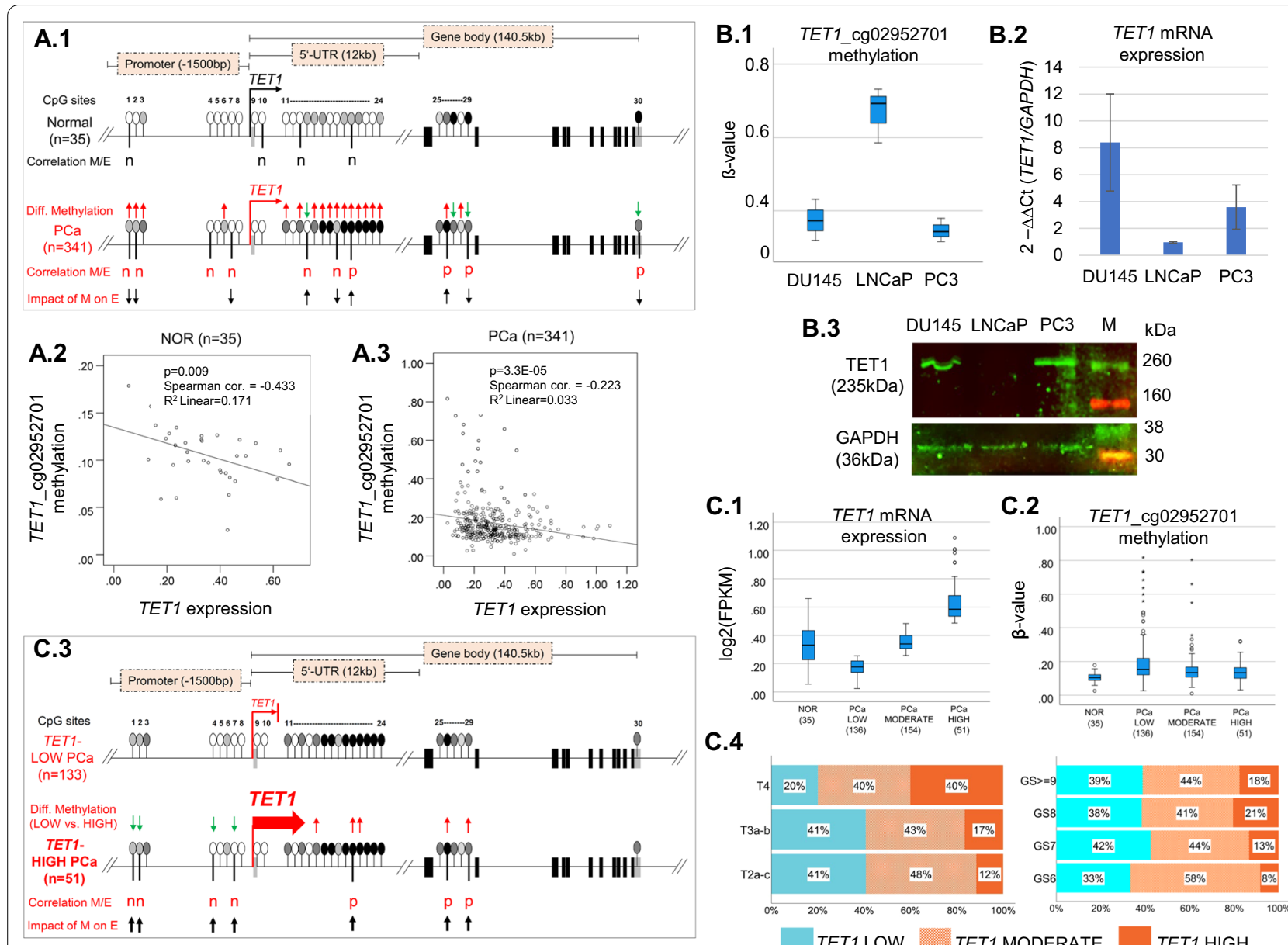

TET1 LOW TET1 MODERATE TET1 HIGH

Fig. 2 Analysis of TET1 promoter methylation and expression in PCa and NOR tissue, and in PCa cell lines. A Based on the transcriptome data (FPKM: fragments per kilobase per million mapped reads) and methylome data ( $\beta$-values) of the Cancer Genome Atlas, we performed correlation analyses between TET1 expression (E) and TET1 methylation (M) at 30 CpG probes in 35 NOR and 341 PCa (A.1, Correlation of methylation with expression (M/E), n: significant negative correlation, p: significant positive correlation, Spearman). Comparison of $\beta$-values at $30 \mathrm{CpG}$ probes in PCa (341) and NOR (35) revealed 22 differentially methylated CpG sites in PCa (A.1, Diff. Methylation, 18 red up-arrows: 18 significantly hypermethylated sites in PCa, four green down-arrows: four significantly hypomethylated sites in PCa, Mann-Whitney $U$ test). The impact of differential methylation at these 22 CpG sites on TET1 expression was assessed according to the correlation of M/E in PCa (A.1, Impact of M on E, down arrows: downregulation, up arrows: upregulation). Methylation at TET1_cg02952701 showed the strongest correlation with TET1 expression in both NOR (A.2) and PCa (A.3); B In LNCaP cells, TET1_cg02952701 was hypermethylated (B.1), and TET1-mRNA and TET1-protein were downregulated (B.2 and B.3). In DU145 and PC3 cells, TET1_cg02952701 was hypomethylated (B.1), and TET1 mRNA and TET1 protein were expressed (B.2 and B.3); C based on TET1 expression, we categorized the PCa cohort into three groups: low (expression $<40$ th percentile, $n=136$ ), moderate ( $\geq 40$ th and $\leq 85$ th percentile, $n=154$ ), and high (> 85th percentile, $n=51)$ (C.1). Accordingly, the TET1-high group showed the least methylation in TET1_cg02952701 and the TET1-low group showed the most methylation (C.2). Comparison of $\beta$-values at 30 CpG probes in TET1-high PCa versus TET1-low PCa revealed nine differentially methylated CpG-sites in TET1-high PCa (C.3, Diff. Methylation, four green arrows: four significantly hypomethylated sites, five red arrows: five significantly hypermethylated sites). The four hypomethylated $\mathrm{CpG}$ sites in the promoter were significantly negatively correlated with TET1 expression and thus, led to TET1 upregulation. Three out of five hypermethylated CpG sites in the 5'-UTR and gene body were significantly positively correlated with TET1 expression and thus also led to TET1 upregulation (C.3, Correlation M/E, $n$ : significant negative correlation, $p$ : significant positive correlation, Spearman; Impact of M on E, up arrows: upregulation). Grouping of PCa by tumor stage and Gleason score revealed that TET1-high PCa occurred more frequently in advanced stages of PCa (C.4)

and NOR, and analyzed it in PCa cell lines with regard to TET1 mRNA and TET1 protein expression. In both PCa and NOR, TET1_cg02952701 located in the TET1 promoter showed the strongest correlation with TET1 expression (Fig. 2A.2, A.3, Additional file 2: Table S3).
Analyses in $\mathrm{PCa}$ cell lines showed that hypermethylation of TET1_cg02952701 in LNCaP cells (Fig. 2B.1) was associated with downregulation of both mRNA and protein expression (Fig. 2B.2, B.3). In comparison, in DU145 and PC3 cells, TET1_cg02952701 was hypomethylated 
(Fig. 2B.1) and TET1 mRNA and protein were expressed (Fig. 2B.2, B.3). Thus, using PCa cell lines, direct causality between TET1 promoter hypomethylation and upregulation of TET1 mRNA and TET1 protein expression was confirmed.

Next, we categorized the TCGA-PCa specimens into three groups according to their TET1 expression levels: TET1-high (expression level above the 85th percentile, $n=51$ ), TET1-low (expression below the 40th percentile, $n=136$ ), and TET1-moderate (expression between the 40th and 85th percentiles, $n=154$ ) (Fig. 2C.1), and analyzed the methylation of TET1_cg02952701. As expected, hypomethylation of TET1_cg02952701 was observed in the TET1-high group, and hypermethylation in the TET1-low group (Fig. 2C.2). We then performed differential methylation analyses considering all 30 TET1 CpG sites in TET1-high versus TET1-low PCa. In TET1-high $\mathrm{PCa}$, four significantly hypomethylated CpG-sites in the TET1-promoter and five significantly hypermethylated CpG-sites in the TET1 $5^{\prime}$-UTR and gene body were detected (Fig. 2C.3, Additional file 2: Table S4 and Additional file 1: Fig. S4). Importantly, the four hypomethylated CpG-sites were significantly negatively correlated with TET1 expression, that is, they contributed to a gain of TET1 expression, and the three hypermethylated $\mathrm{CpG}$ sites were significantly positively correlated with TET1 expression, that is, they also contributed to a gain of TET1 expression (Fig. 2C.3 and Additional file 2: Table S3). Thus, seven differentially methylated CpGsites in TET1 caused an overexpression of TET1 in PCa. Further analyses showed that TET1-high PCa specimens were more frequently detected among $\mathrm{PCa}$ with higher Gleason scores (Gleason 6: 8.4\%, Gleason 7: 13.3\%, Gleason 8: $20.5 \%$, Gleason $\geq 9: 17.6 \%$ ) and higher tumor stages (T2a-c: 11.5\%, T3a-b: 16.6\%, T4: 40\%) (Fig. 2C.4).

\section{Identification of 626 TET1-coactivated genes in PCa}

To investigate the impact of cells and cell clusters in $\mathrm{PCa}$ expressing TET1 and TET1 at high levels, we performed genome-wide correlation and gene regulatory network analyses using transcriptome and methylome data of PCa patients listed in TCGA (Fig. 3). Based on transcriptome data, we calculated the Pearson's correlation coefficients between TET1 expression and the expression values of all 19,252 protein-coding genes. Of 7533 genes exhibiting a statistically significant positive correlation to TET1 expression $(p<0.05), 626$ showed particularly strong correlation (Pearson's $r>0.5, p<0.0001$ ). These 626 genes were referred to as TET1-coactivated genes and were subjected to further detailed bioinformatics studies (Fig. 3). TET1 expression was not correlated to AMACR expression (Pearson's $r=0.01, p=0.8$ ). As TET1 is a 5-methylcytosine dioxygenase, and demethylation of gene promoters leads to increased expression of the respective mRNA, we also calculated the Spearman's correlation coefficients for the promoter methylation of 626 TET1-coactivated genes and TET1 expression. Of 618 genes represented in the Illumina $450 \mathrm{~K}$ array, 594 possessed $\mathrm{CpG}$ sites in their promoters (sequences within 1500 and $200 \mathrm{bp}$ of the transcriptional start siteTSS1500 and TSS200, respectively-were considered). In 279 out of 594 genes, the promoter was hypomethylated when TET1 expression was increased, and in TET1-high PCa these gene promoters were significantly less methylated than in TET1-low PCa. In 235 of those 279 genes, promoter methylation was significantly negatively correlated with the gene's expression, that is, the hypomethylation of the promoter led to increased gene expression.

\section{Upregulation of TET1 in PCa strongly correlates with promoter demethylation and enhanced expression of genes encoding zinc-finger transcription factors} According to the Human Transcription Factor (TF) databank [30], 161 out of 626 TET1-coactivated genes in PCa are identified TFs. Remarkably, 133 out of 161 TET1coactivated TFs $(82.6 \%)$ belong to the group of zinc-finger TFs (Fig. 4) and were significantly enriched for the GO "Molecular function" terms "DNA-binding transcription activator activity" (23/133, $p=5.0 \mathrm{e}-08)$ and "DNA-binding transcription repressor activity" (15/133, $p=1.0 \mathrm{e}-07)$. Among the top 30 TET1-coactivated TFs, we found primarily stem cell- and cancer-associated TFs, for example, RFX7 (an X-box-recognizing TF involved in cellular specialization and differentiation), REST (a Kruppel-type TF involved in the regulation of stem cell pluripotency), ZNF292 (a growth hormone-dependent TF with tumor suppressor activity), ARID2 (an AT-rich interactive domain-containing TF involved in embryonic patterning and cell lineage gene regulation), ZXDB (an $\mathrm{X}$-linked TF promoting MHC gene expression), NR2C2 (a nuclear hormone receptor involved in aging-associated diseases), and SP1 (a Kruppel-like TF involved in cell growth and differentiation) (Fig. 4A). Importantly, the genes encoding the top 30 TET1-coactivated TFs all possess at least one promoter CpG site that was significantly demethylated when TET1 was significantly upregulated, and all these genes possessed significantly hypomethylated promoters in TET1-high PCa versus TET1-low PCa (Fig. 4B and Additional file 2: Table S6). In total, we identified 68 TFs in PCa whose gene promoters were significantly hypomethylated when TET1 was significantly upregulated (Additional file 2: Table S6).

Next, using JASPAR 2020 we analyzed the binding motifs and binding scores of TET1-coactivated TFs in the TET1 promoter and in the promoters of 626 TET1-coactivated genes (TSS1500 was considered). Of 161 TFs, 21 


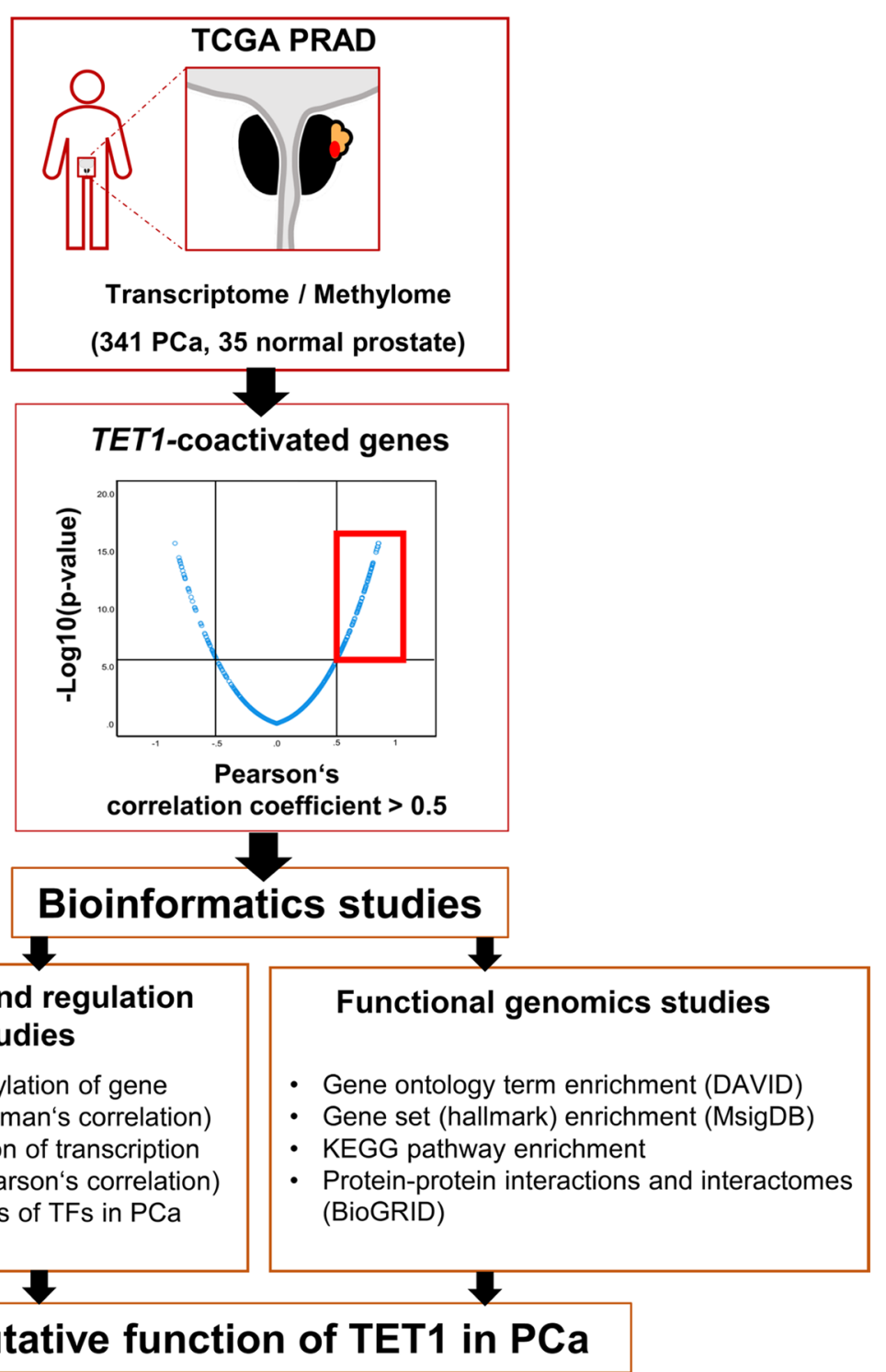

Fig. 3 Work plan and methodology for creating a TET1-correlated gene regulation network and for functional genomics. The transcriptome and methylome data in the Cancer Genome Atlas (TCGA) generated from 341 PCa, were applied. TET1-coactivated genes were evaluated using Pearson's correlation, and genes having $r>0.5$ were considered in bioinformatics studies

were listed in JASPAR 2020 and could be analyzed. In the TET1 promoter, TF-BSs were found for all 21 analyzed TFs (Additional file 2: Table S5). Segments in the TET1 promoter that were found to be significantly hypomethylated in TET1-high PCa and hence decisive for TET1upregulation (Fig. 2C.3 and Additional file 2: Table S4) exhibited TF-BSs for RFX7, NR2C2, SP1, CREB1, MGA, SMAD5, ZBTB6, ZSCAN9, and ZNF354C (Additional file 2: Table S4). The vast majority of 626 TET1-coactivated genes also exhibited TF-BSs for 19 out of 21 TFs (Additional file 2: Table S5). The mean number of TFBSs per gene promoter varied from a few (1 to 10), for example, in ZBTB26, ZKSCAN1, ZKSCAN29, SMAD5, POU2F1, ZBTB6, SP4, ZNF148, EHF, GABPA, CREB1, RREB1, and REST, to a great many $(>10)$, for example, in ZNF384, NR2C2, ZNF354C, RFX7, MGA, CLOCK, SP3, and SP1 (Additional file 2: Table S5). Nine out of 21 TET1-coactivated TFs, namely RFX7, SP1, SP3, SP4, POU2F1, ZBTB6, REST, CLOCK, and ZSCAN29, possessed CGs in their binding motifs and hence were sensitive to CpG-methylation and demethylation (Additional file 1: Fig. S5).

Many TFs of different families do not work alone and form complex homotypic or heterotypic interactions 


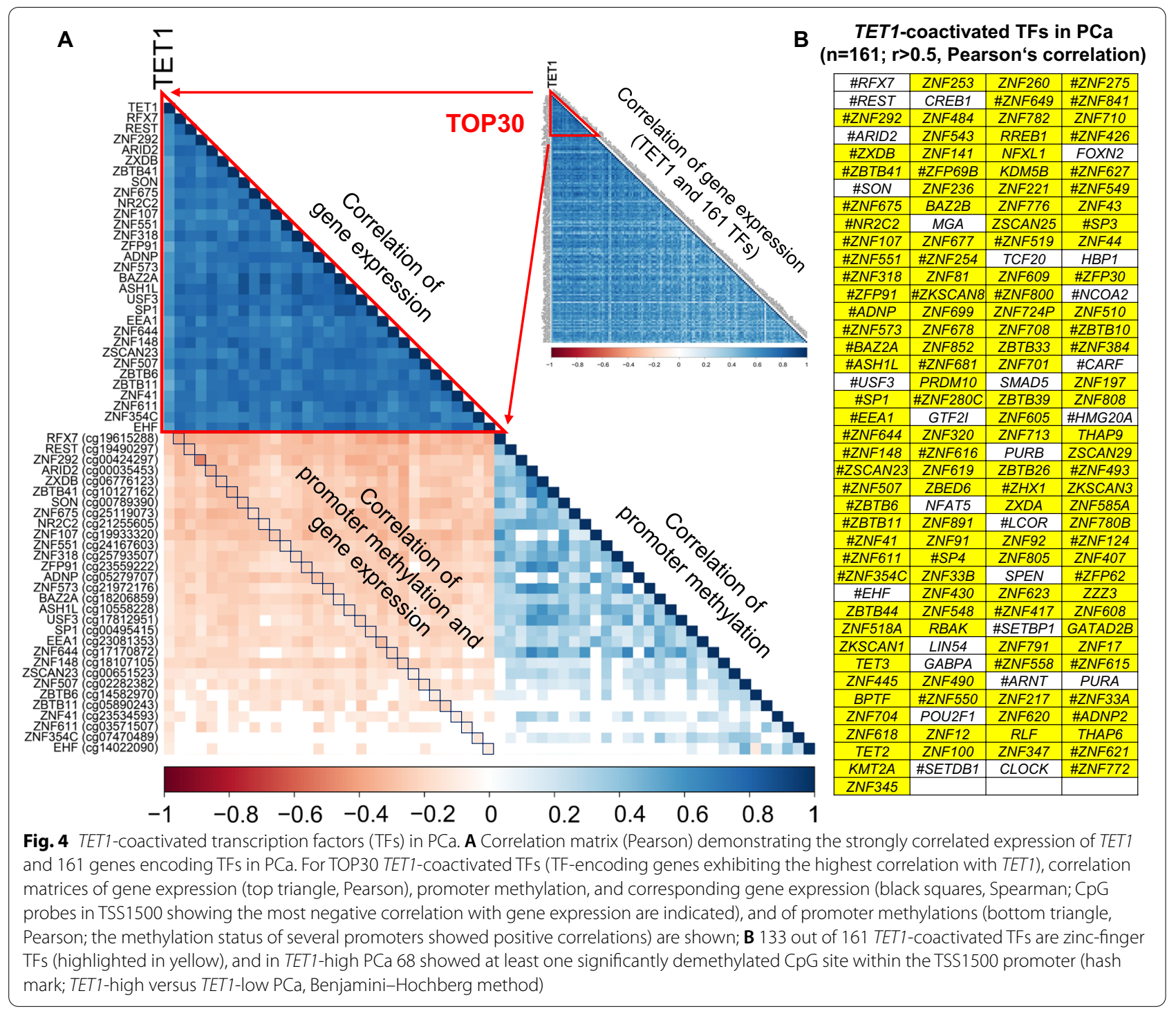

through dimerization [38]. Therefore, we analyzed the protein-protein interactions (PPI) and PPI networks (interactomes) of 161 TET1-coactivated TFs. Interactome analyses centered on $21 \mathrm{TFs}$ that were available in JASPAR 2020 and showed true binding sites in the TET1 promoter and promoters of TET1-coactivated genes. Two interactomes, one SP1-centered and one CREB1-centered, were revealed (Additional file 1: Fig. S6). SP1, a zinc-finger transcription activator binding to GC-rich motifs, showed a direct interaction with six TFs (POU2F1, GABPA, ARNT, SP3, REST, and PURA) (Additional file 1: Fig. S6A.1) and an indirect interaction (i.e., through a bridge TF) with another 33 TFs (Additional file 1: Fig. S6A.2). CREB1, a transcription activator that binds to the cAMP response elements in many mammalian and viral promoters, showed a direct interaction with three TFs (ZHX1, POU2F1, and ZNF92) (Additional file 1: Fig. S6B.1) and an indirect interaction with a further 31 TFs (Additional file 1: Fig. S6B.2).

\section{TET1-coactivated genes in PCa point to a cumulative gain of chromatin remodeling and mitotic activities}

To characterize the functional features shared by 626 TET1-coactivated genes in $\mathrm{PCa}$, we performed functional genomics studies (Fig. 3). GO analyses revealed a significant enrichment of biological processes primarily responsible for chromatin remodeling, such as "covalent chromatin modification," "histone modification," and "peptidyl-lysine modification" (Fig. 5A and Additional file 2: Table S7). In total, 78 genes encoding epigenetic modifiers were activated in PCa together with TET1 (Additional file 2: Table S7 and Additional file 1: Fig. S7). Moreover, significant enrichments of biological processes involved in regulating DNA metabolism and 


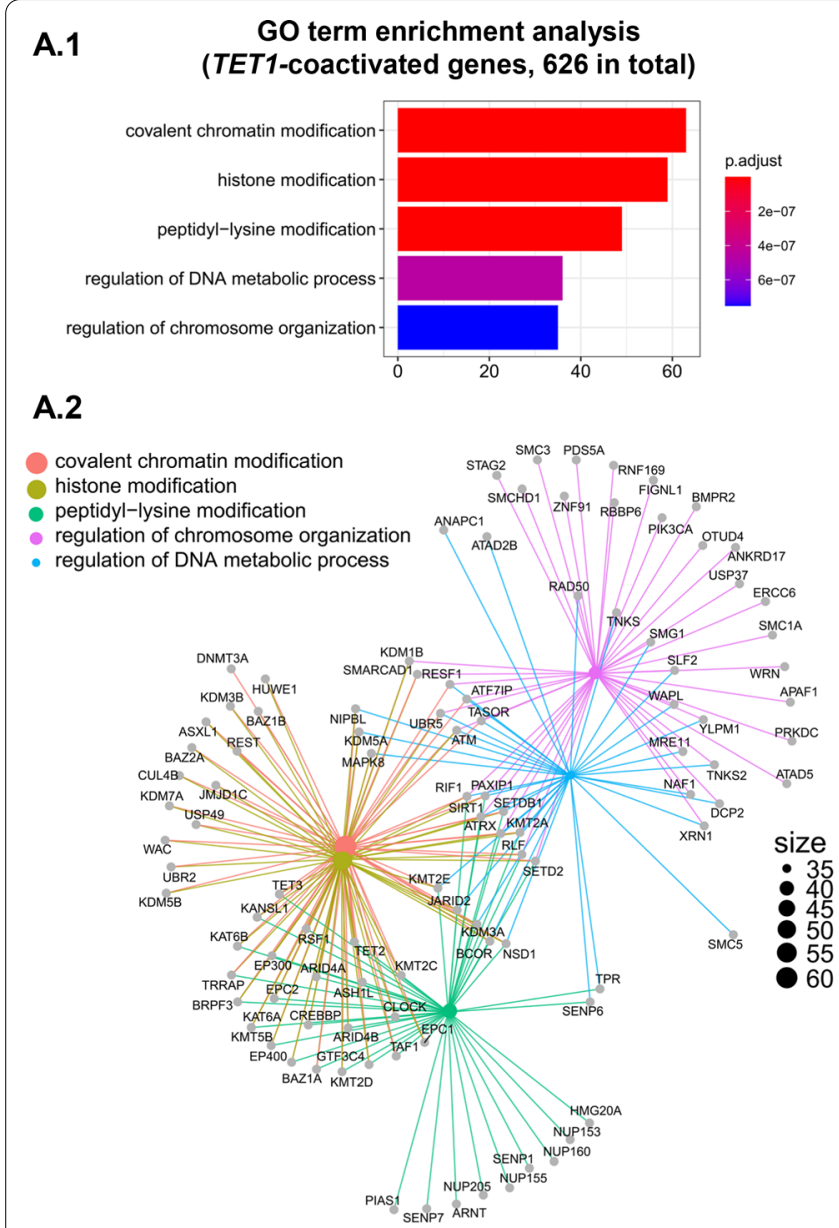

B.1

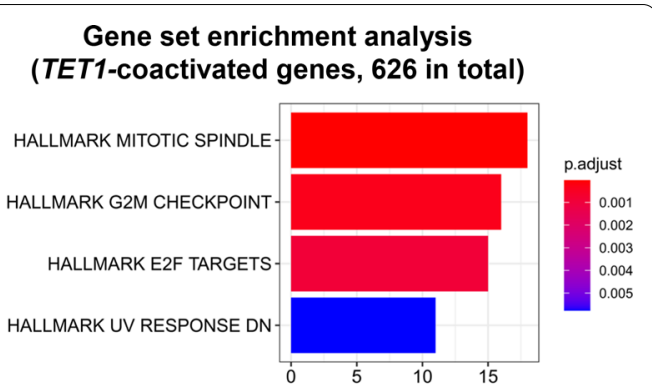

B.2

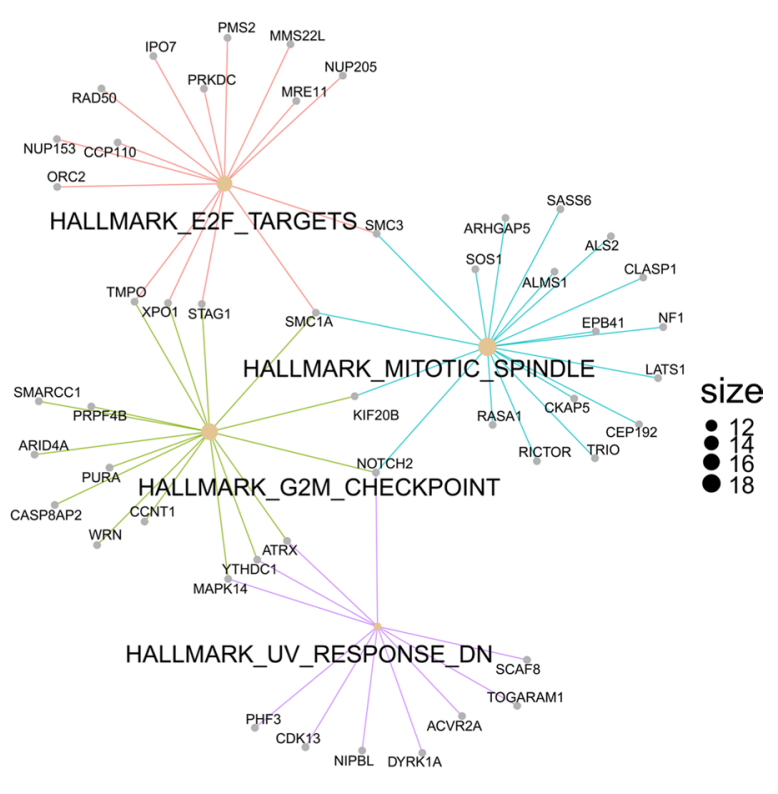

Fig. 5 Functional characterization of TET1-coactivated genes in PCa. A Gene ontology (GO) term enrichment analysis of 626 TET1-coactivated genes (A.1) and network plot of enriched terms depicting the linkages of individual genes and GO terms (A.2) (adjusted $p$ values and numbers of included genes are given); $\mathbf{B}$ gene set (hallmark) enrichment analysis of 626 TET1-coactivated genes (B.1) and network plot of enriched terms depicting the linkages of individual genes and hallmarks (B.2) (adjusted $p$ values and numbers of included genes are given)

chromosome organization were also detected (Fig. 5A). Among TET1-coactivated genes, we found TET2, TET3, and DNMT3A. In both NOR and $\mathrm{PCa}$, strong positive correlations between the expression of TET1, TET2, TET3, and DNMT1 were found (Additional file 1: Fig. $\mathrm{S} 8)$. However, only in $\mathrm{PCa}$, strong positive correlations were also found between TET1 and DNMT3A and DNMT3B expression (Additional file 1: Fig. S8). Further, GSEA of "Hallmark gene sets" on 626 TET1-coactivated genes showed a significant enrichment of mitotic and DNA replication hallmarks, in particular "mitotic spindle," "G2M checkpoint," and "E2F targets" (Fig. 5B and Additional file 2: Table S7). In total, 41 genes encoding mitotic factors were activated in $\mathrm{PCa}$, together with TET1 (Additional file 2: Table S7). In order to examine, whether the P53-pathway is affected alongside with the E2F targets, we performed a GSEA of "Curated gene sets," in particular gene sets representing canonical pathways. Among 626 TET1-coactivated genes, we found significant enrichments for pathways related to regulation of P53 activity by phosphorylation and methylation, and to P53-hypoxia (Additional file 2: Table S7). Moreover, GSEA of "Oncogenic signature gene sets" representing cellular pathways often dysregulated in cancer [35] on 626 TET1-coactivated genes showed highly significantly enrichments for gene sets related to Kirsten rat sarcoma viral oncogene homolog (KRAS) and TANK Binding Kinase 1 (TBK1), Placental Growth Factor (PGF), Janus Kinase 2 (JAK2), Catenin Beta 1 (CTNNB1) and Vascular Endothelial Growth Factor A (VEGFA) (Additional file 1: Fig. S9 and Additional file 2: Table S8).

Next, we performed TCGAanalyze_SurvivalKM, a univariate Kaplan-Meier (KM) survival analysis on 626 TET1-coactivated genes in PCa (Fig. 6, Additional file 2: Table S9). In total, expression of 35 genes showed a significant impact on survival (Additional file 2: Table S9). 

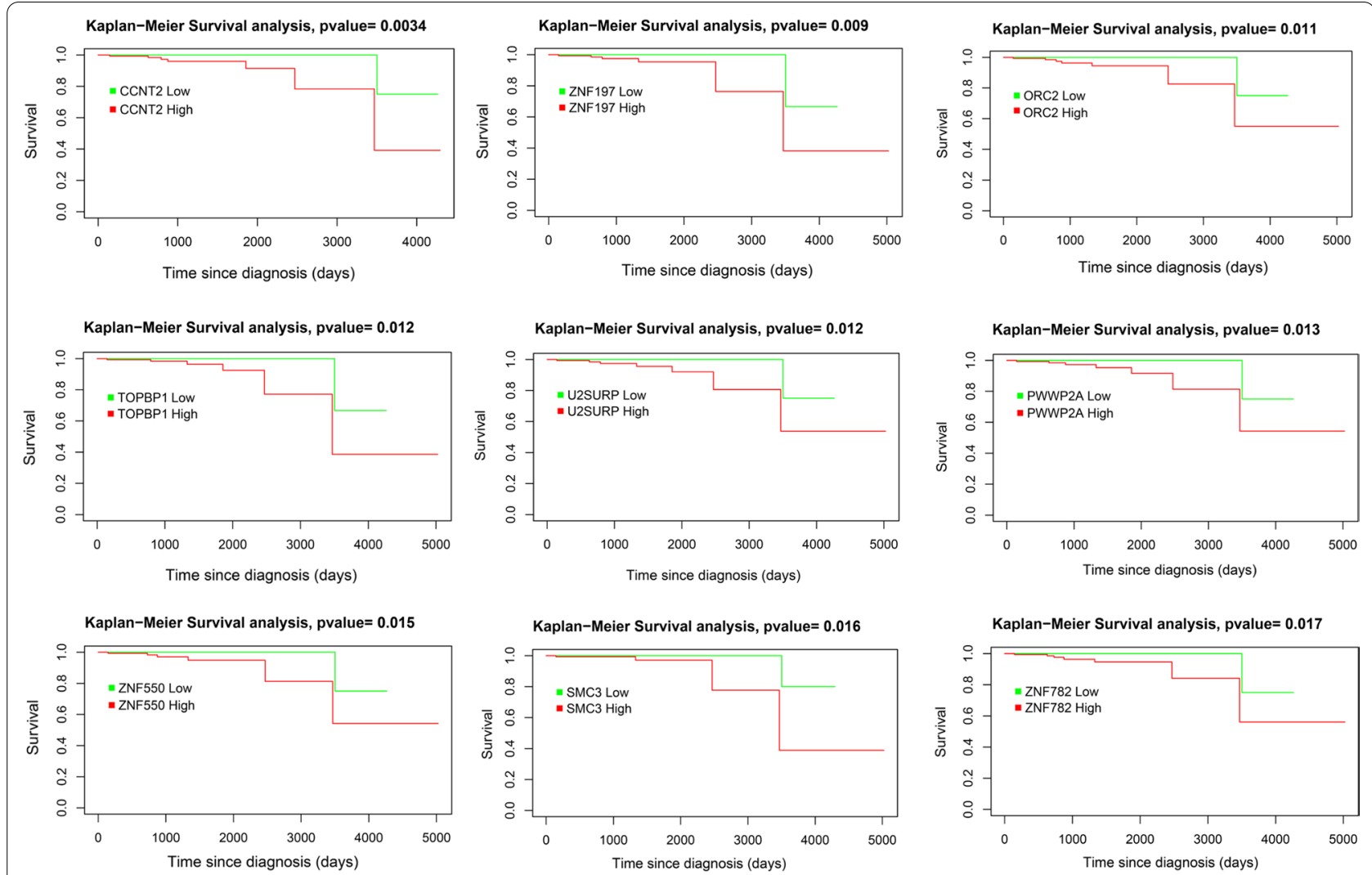

Fig. 6 Kaplan-Meier survival analysis on TET1-coactivated genes in PCa. In total, expression of 35 out of 626 TET1-coactivated genes was significantly associated with survival. Survival curves of top 9 genes are shown $(p$ value $<0.02)$

Survival curves of top 9 candidates (CCNT2, ZNF197, ORC2, TOPBP1, U2SURP, PWWP2A, ZNF550, SMC3 and ZNF782) are shown in Fig. 6 ( $p$ value $<0.02$ ).

Analyzing publicly available TET1- and Tet1-ChIP-seq data, we discovered a strong similarity of MSigDB-hallmark- and GO-enrichment profiles in $\mathrm{PCa}$ and mouse trophoblast stem cells (mTSCs) [9, 10]. In mTSCs, 2691 gene promoters (defined as TSS1500) exhibited Tet1 binding sites (Fig. 7). A parallel analysis of TET1-coactivated genes in $\mathrm{PCa}$ and genes exhibiting Tet1 binding sites in mTSCs revealed an enrichment of nearly the same GO terms (primarily associated with chromatin modification) and same gene sets (primarily associated with mitosis) (Fig. 7).

\section{TET1-correlated activation of ZNF antiviral genes in PCa}

To explore the pathways activated in TET1-high $\mathrm{PCa}$, we performed KEGG pathway analyses on 626 TET1coactivated genes (Additional file 1: Fig. S10). We found that the "herpes simplex virus 1 infection" (HSV-1 infection) pathway was the most significantly enriched one (Additional file 1: Fig. S10A and S10B). In total, 62 genes encoding ZNF antiviral proteins (ZAPs) showed a strong positive correlation to TET1 expression and were significantly upregulated in TET1-high PCa (Additional file 1: Fig. S10C and Additional file 2: Table S10). Besides the genes encoding ZAPs, we also detected five genes encoding signaling molecules involved in the immune response to cancer, namely TRAF6, EIF2AK2, PIK3CA, APAF1, and POU2F2 (Additional file 1: Fig. S10C). Therefore, to assess the impact of viral infections in $\mathrm{PCa}$, we analyzed $34 \mathrm{PCa}$ and 16 benign prostate hyperplasia (BPH) samples with regard to different viral infections, including the herpesviruses HCMV, EBV and HSV-1/-2, and the polyomaviruses BK and JC (Additional file 2: Table S11). We detected infections with HCMV in 11/34 (32.4\%) PCa and $3 / 16(18.8 \%) \mathrm{BPH}$, and EBV in 5/34 (14.7\%) PCa and 1/16 (6.3\%) BPH (Additional file 2: Table S11). No infections with HSV-1/-2 or JC-/BK-viruses were found in $\mathrm{PCa}$ and $\mathrm{BPH}$.

Furthermore, KEGG pathway analyses also revealed a significant enrichment of genes involved in "signaling pathway regulating pluripotency of stem cells" (Additional file 1: Fig. S10D and Additional file 2: Table S10). The expression of several genes encoding regulators of stem cell pluripotency, including PI $3 K, A C V R 1 / 2$, 


\section{TET1-coactivated genes}

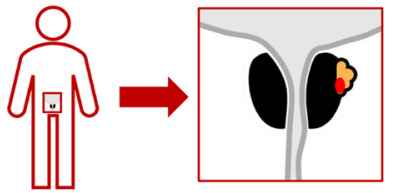

TET1-HIGH expressing PCa
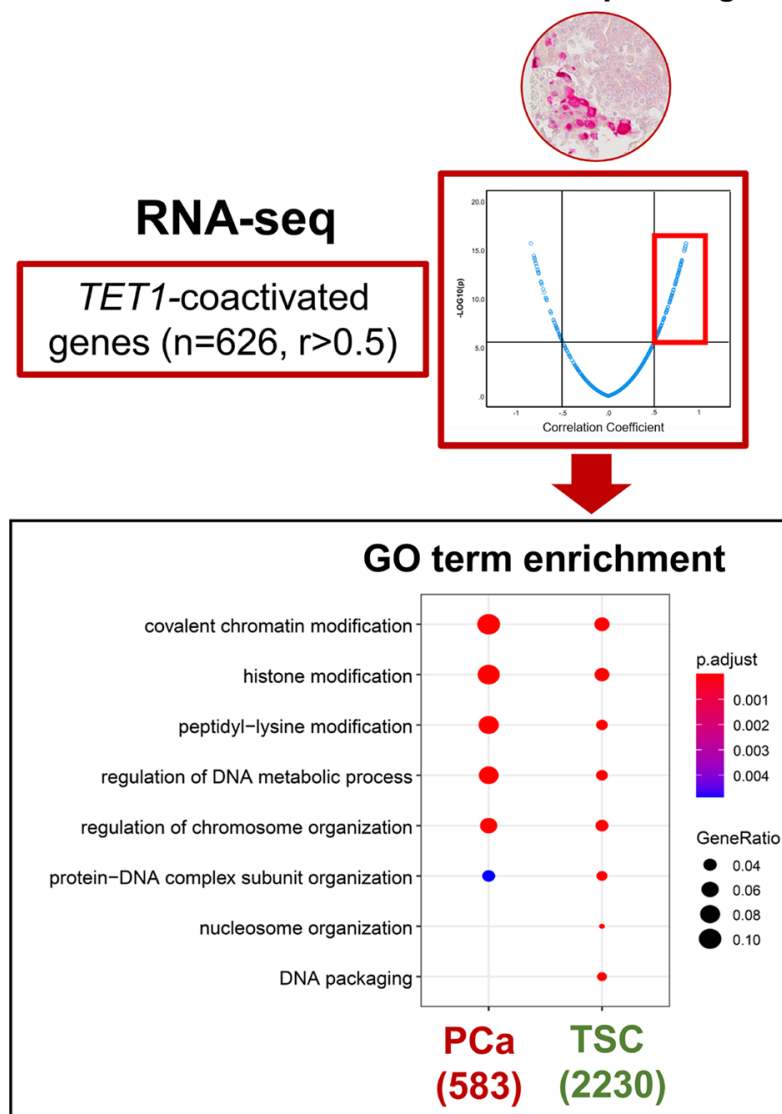

Tet1-bound gene promoters

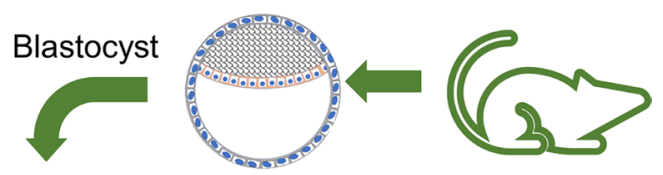

Trophoblast stem cells (TSC)
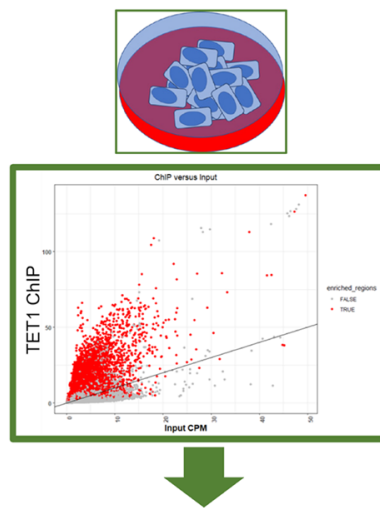

Tet1-CHIP-seq

Tet1-bound

promoters $(n=2691)$

Fig. 7 Comparison of TET-coactivated genes in PCa and Tet1-bound genes in mouse trophoblast stem cells. TET1-coactivated genes in PCa (626) were analyzed in parallel to genes exhibiting Tet1-binding sites within the TSS1500-promoter (2691) with regard to gene ontology (GO) term enrichment (bottom left), and gene set enrichment (bottom right; MsigDB: Molecular Signatures Database). The results showed enrichment of nearly exactly the same GO terms (primarily associated with chromatin modification) and gene sets (primarily associated with mitotic division) in the two groups

BMPR1/2, GSK3B, p38, SMARCAD1, SETDB1, JARID2, $K A T 6 A, R E S T$, and RIF1 showed a strong positive correlation with TET1 expression in PCa and were coactivated in TET1-high PCa (Additional file 1: Fig. S10D).

\section{Discussion}

Cancer cells, also within a tumor, may be heterogenous and very with regard to their molecular profiles and strategies to achieve a growth advantage. The identification of self-renewing cancer stem cells driving tumor growth and metastasis is the primary objective of attempts to discover biomarkers in aggressive PCa. As a key regulator of stem cell pluripotency, and being dysregulated in a wide range of human malignancies, TET1 is a highly relevant candidate. As yet, TET1 has been poorly investigated in PCa. Prior studies on TET1 have analyzed a relatively small number of primary PCa specimens [28, $29,39]$ or have been limited to cell culture experiments [40, 41]. TET1 has been shown to suppress PCa invasion by activating tissue inhibitors of metalloproteinases [28]. TET1 protein levels are decreased in $\mathrm{PCa}$, and low TET1 mRNA levels are significantly associated with worse metastasis-free survival [29]. However, distinct activating and repressive functions of TET1-mediated 
transcriptional regulation could also be demonstrated in PCa [39].

In this context, we performed a large-scale IHC study, analyzing a large number of tissue samples by IHC using both conventional slides (NOR and PCa specimens originating from 50 PCa patients) and TMA (669 single NORTMA spots and 1371 single PCa-TMa spots originating from $371 \mathrm{PCa}$ patients). These two complementary methods allowed us to get a comprehensive and detailed view on TET1 expression in the prostate. Our data show that in normal prostate, TET1 was exclusively expressed in CK903-positive basal cells, which were scattered in the basal epithelium known to contain the prostate stem cells $[12,15]$. In contrast, in $\mathrm{PCa}$, where the basal cell layer was often destroyed, we could frequently detect TET1 expression in AMACR-positive cancer cells. Remarkably, TET1-expressing PCa cells often occurred in big clusters, and these clusters were observable at all tumor stages. However, among NOR-TMA spots, some few foci also possessed TET1-high-expressing cells, but the frequency of occurrence was sixfold lower than among PCaTMA spots. Based on their TET1-expression profiles, PCa specimens could be stratified into three subgroups (TET1-high, moderate, and negative), and TET1-high $\mathrm{PCa}$ specimens exhibiting numerous clearly defined TET1-positive cell clusters accounted for $17.5 \%$ of all PCa. Regulation of DNA demethylation and, hence, the transcriptional activity of pluripotency genes is the key function of TET methyl-cytosine dioxygenases including TET1, and is essential for the maintenance of stem cell self-renewal capacity, as shown in various mouse and human stem cell types [42]. According to our results, in the normal prostate TET1 may be involved in maintaining basal cell stemness, and in $\mathrm{PCa}$ (more precisely in TET1-expressing cell clusters/colonies), it may be aberrantly upregulated and enforce oncogenic properties. Much evidence indicates that $\mathrm{PCa}$ arises from pluripotent CSCs that possess a certain level of plasticity and are major determinants of tumor development and progression [15]. The multitude of CSC surface and intracellular markers detected in PCa, for example, c-kit, CD133, CD44, $\alpha 2 \beta 1$ integrin, CXCR4, Sox2, Oct3/4, Nanog, c-myc, and Klf4 [15], emphasizes the molecular and biological diversity of CSCs in PCa. Our results suggest that TET1 expression could be characteristic of proliferating CSCs in PCa.

In our study, IHC revealed that TET1 may reside in the nucleus as well as in the cytoplasm in both $\mathrm{PCa}$ and the normal basal epithelium. Similar subcellular localization of TET1 has been observed in ovarian endometriotic lesions, gastric and ovarian cancers, hippocampal neurons, and gliomas [25, 43-46]. Some studies have addressed the impact of the nuclear/ cytoplasmic TET1 ratio on genome-wide DNA methylation levels, but the results have been inconclusive. In cell culture, under low oxygen conditions, an observed increase in the nuclear/cytoplasmic TET1 ratio has been suggested to lead to an increase in $5 \mathrm{hmC} \mathrm{[10].}$ However, complete cytoplasmic TET1 translocation did not lead to significant alteration of $5 \mathrm{hmC}$-levels [47]. O-GlcNAc transferase has been reported to physically and functionally interact with TETs and be responsible for their subcellular localization [48].

Next, by comparing TET1-high and -low PCa, we revealed in TET1-high specimens significantly demethylated CpG-sites in TET1 promoter, which significantly correlated with an increased TET1-mRNA and TET1-protein expression. Remarkably, TET1-high PCa were more frequently detected among advanced tumor stages. In order to characterize the molecular profile of TET1-high PCa, we evaluated the expression of other genes for correlation with TET1 expression. The correlation threshold set at 0.5 (Pearson's $r>0.5$ ) provided a conclusive balance between strong correlation and a sufficiently informative number of TET1-correlated genes. Among 626 genes upregulated together with TET1 in PCa, significant enrichments were found primarily for genes encoding chromatin remodeling and mitotic factors. Furthermore, specific oncogenic signature gene sets and pathways were enriched too. Among TET1-coactivated genes, we also detected 161 encoding TFs, with a particular dominance of ZNF-TFs $(133 / 161)$. All 21 TFs that were analyzed in detail had significant binding sites in the TET1 promoter as well as in the promoters of the vast majority of TET1-coactivated genes. It is very likely that all 626 TET1-coactivated genes possess TET1-BSs in their promoters, as exactly the same GO terms, and gene hallmarks were enriched when Tet1-binding promoters in mouse TSCs were analyzed [9], and many exhibited significantly hypomethylated promoters in TET1-high in comparison with TET1-low PCa. These findings point to an orchestrated activation of TET1 and these 626 genes, and together with our IHC observations strengthen the hypothesis that AMACR+/TET1+ cancer cells and cell clusters in $\mathrm{PCa}$ may constitute proliferating cell colonies and represent a specific cell entity with stem cell attributes and an oncogenic signature. There are a number of reports demonstrating the oncogenic potential of TET1, in particular in ovarian and breast cancer [49-51]. In triple-negative breast cancer, which is one of the most hypomethylated cancers, TET1-mediated hypomethylation activates oncogenic signaling [27]. The authors suggested that TET1, as a potential oncogene, could serve as a druggable target for therapeutic intervention [27]. Our results support this idea and 
furthermore suggest that TET1 might be a good candidate for tracing mitotically active cells with stem cell attributes in PCa.

It is known that the prostate normally contains abundant intracellular zinc and that dysregulation of zinc homeostasis, in particular zinc loss, is a hallmark of PCa development [52]. Interestingly, in addition to 133 ZNF-TFs, we found 62 ZAPs and several signaling molecules involved in the antiviral immune response to be activated at gene level together with TET1 and enriched for the KEGG-pathway "HSV-1 infection." Our findings emphasize the impact of zinc-dependent proteins and their dysregulation in $\mathrm{PCa}$, and indicate a possible herpesvirus-mediated path of TET1 activation. However, we did not detect HSV-1/2-infections, but found twice as many infections with other herpesviruses HCMV and EBV in PCa as in BPH. Reactivation of HCMV may occur in previously immunocompetent patients postoperatively after chemo- or radiotherapy, and is associated with poor outcomes, including other infections and mortality [53]. Zinc is an essential trace element crucial for immune function, can influence antiviral immunity, and is discussed as possibly favorable additive against viral infections such as HSV and the common cold [54]. Thus, zinc-mediated therapeutics may also be an effective approach to PCa prevention and treatment.

In conclusion, our results suggest that in PCa, TET1expressing cells and cell colonies may be proliferating tumor cells with a distinct oncogenic signature. Our study contributes to a better understanding of TET1 function in human malignancies and underlines its potential for marker development.

\section{Abbreviations}

TET1: Ten-eleven translocation family member 1; PCa: Prostate cancer; BPH: Benign prostate hyperplasia; NOR: Normal prostate; CSCs: Cancer stem cells; TMA:Tissue microarray; CK903: Cytokeratin 903; AMACR: Alpha-methylacylCoA racemase; ZNF: Zinc finger; ZAP: Zinc finger antiviral protein; TF: Transcription factor; TCGA: The Cancer Genome Atlas; DAVID: Database for Annotation, Visualization and Integrated Discovery; MSigDB: Molecular Signatures Database; GSEA: Gene set enrichment analysis; KEGG: Kyoto Encyclopedia of Genes and Genomes; PPI: Protein-protein interaction; HCMV: Human cytomegalovirus; EBV: Epstein-Barr virus; HSV: Herpes simplex virus; RT-qPCR: Reverse transcription quantitative polymerase chain reaction.
\end{abstract}

\section{Supplementary Information}

The online version contains supplementary material available at https://doi. org/10.1186/s13148-021-01201-7.

Additional file 1. Supplementary Figures.

Additional file 2. Supplementary Tables.

\section{Acknowledgements}

We thank Mrs. Tania Bloch, Mrs. Kerstin Wilhelm, and Mrs. Barbara Froehlich for technical assistance in collection of prostate tissue samples and biobanking at the Clinic of Urology, Pediatric Urology and Andrology of the
Justus-Liebig-University Giessen. We thank the Tumor and Normal Tissue Bank of the UniversitätsKrebsCentrum Dresden for providing the prostata tissue samples.

\section{Authors' contributions}

TD and US designed the study, analyzed and interpreted the data; LC performed IHC; DS and SG assessed the histology of prostate tissue samples; SF, GBB and LCH assembled and characterized prostate TMA; NB-S performed scanning and digitalization of TMA; HS performed virus PCR assays; FW provided resources; TD performed bioinformatics analyses; TD and US prepared the figures and wrote the paper. All authors read and approved the final manuscript.

\section{Funding}

Open Access funding enabled and organized by Projekt DEAL. The study was supported by a Research Grant of the University Medical Center Giessen and Marburg to T. D. and F.W. (UKGM, Project Nr. 6/2012GI), and by the German Research Foundation (DFG), in the frame of the IRTG 1871 "Molecular pathogenesis of male reproductive disorders" (Grant to F.W. and U.S., Project 5).

Availability of data and materials

Not applicable.

\section{Declarations}

\section{Ethics approval and consent to participate}

The study was approved by the ethics committee of the Medical Faculty, Justus-Liebig-University Giessen, Germany (Ethics Vote: 49/05), and all patients gave their written consent to participate in the study.

\section{Consent for publication \\ Not applicable.}

\section{Competing interests}

The authors declared that they have no competing interests.

\section{Author details}

${ }^{1}$ Clinic of Urology, Pediatric Urology and Andrology, Justus-Liebig-University Giessen, Giessen, Germany. ${ }^{2}$ Working Group Epigenetics of Urogenital System, Clinic of Urology, Pediatric Urology and Andrology, Justus-Liebig-University Giessen, Giessen, Germany. ${ }^{3}$ Institute of Medical Virology, Justus-Liebig-University Giessen, Giessen, Germany. ${ }^{4}$ Institute of Pathology, Justus-Liebig-University Giessen, Giessen, Germany. ${ }^{5}$ Department of Urology, University Hospital and Faculty of Medicine, Technische Universität Dresden, Dresden, Germany. ${ }^{6}$ Institute of Neuropathology, Justus-Liebig-University Giessen, Giessen, Germany. ${ }^{7}$ Institute of Pathology, University Hospital and Faculty of Medicine, Technische Universität Dresden, Dresden, Germany. ${ }^{8}$ Division of Endocrinology, Diabetes, and Bone Diseases, Department of Medicine III and University Center for Healthy Aging, Technische Universität Dresden, Dresden, Germany.

Received: 9 September 2021 Accepted: 22 November 2021 Published online: 29 November 2021

References

1. An J, Rao A, Ko M. TET family dioxygenases and DNA demethylation in stem cells and cancers. Exp Mol Med. 2017;49:e323-e323.

2. Verma N, Pan H, Doré LC, Shukla A, Li QV, Pelham-Webb B, et al. TET proteins safeguard bivalent promoters from de novo methylation in human embryonic stem cells. Nat Genet. 2018;50:83-95.

3. Ficz G, Branco MR, Seisenberger S, Santos F, Krueger F, Hore TA, et al. Dynamic regulation of 5-hydroxymethylcytosine in mouse ES cells and during differentiation. Nature. 2011;473:398-402.

4. Williams K, Christensen J, Pedersen MT, Johansen JV, Cloos PAC, Rappsilber J, Helin K. TET1 and hydroxymethylcytosine in transcription and DNA methylation fidelity. Nature. 2011;473:343-8.

5. Wu H, D'Alessio AC, Ito S, Xia K, Wang Z, Cui K, et al. Dual functions of Tet1 in transcriptional regulation in mouse embryonic stem cells. Nature. 2011;473:389-93. 
6. Koh KP, Yabuuchi A, Rao S, Huang Y, Cunniff K, Nardone J, et al. Tet1 and Tet 2 regulate 5 -hydroxymethylcytosine production and cell lineage specification in mouse embryonic stem cells. Cell Stem Cell. 2011:8:200-13.

7. Shimozaki K. Ten-eleven translocation 1 and 2 confer overlapping transcriptional programs for the proliferation of cultured adult neural stem cells. Cell Mol Neurobiol. 2017;37:995-1008.

8. Yang R, Yu T, Kou X, Gao X, Chen C, Liu D, et al. Tet1 and Tet2 maintain mesenchymal stem cell homeostasis via demethylation of the P2rX7 promoter. Nat Commun. 2018;9:1-14.

9. Chrysanthou S, Senner CE, Woods L, Fineberg E, Okkenhaug H, Burge $S$, et al. A critical role of TET1/2 proteins in cell-cycle progression of trophoblast stem cells. Stem Cell Rep. 2018;10:1355-68.

10. Senner CE, Chrysanthou S, Burge S, Lin H-Y, Branco MR, Hemberger M. TET1 and 5-hydroxymethylation preserve the stem cell state of mouse trophoblast. Stem Cell Rep. 2020;15:1301-16.

11. Tie G, Yan J, Khair L, Tutto A, Messina LM. Hypercholesterolemia accelerates the aging phenotypes of hematopoietic stem cells by a Tet1-dependent pathway. Sci Rep. 2020;10:1-11.

12. de Marzo AM, Meeker AK, Epstein JI, Coffey DS. Prostate stem cell compartments. Am J Pathol. 1998;153:911-9.

13. Nguyen LV, Vanner R, Dirks P, Eaves CJ. Cancer stem cells: an evolving concept. Nat Rev Cancer. 2012;12:133-43.

14. Valent P, Bonnet $\mathrm{D}$, de Maria R, Lapidot T, Copland M, Melo JV, et al. Cancer stem cell definitions and terminology: the devil is in the details. Nat Rev Cancer. 2012;12:767-75.

15. Moltzahn F, Thalmann GN. Cancer stem cells in prostate cancer. Transl Androl Urol. 2013;2:242-53.

16. Harris KS, Kerr BA. Prostate cancer stem cell markers drive progression, therapeutic resistance, and bone metastasis. Stem Cells Int. 2017:2017:1-9.

17. Kyjacova L, Hubackova S, Krejcikova K, Strauss R, Hanzlikova H, Dzijak $\mathrm{R}$, et al. Radiotherapy-induced plasticity of prostate cancer mobilizes stem-like non-adherent, Erk signaling-dependent cells. Cell Death Differ. 2015;22:898-911

18. Tsao T, Beretov J, Ni J, Bai X, Bucci J, Graham P, Li Y. Cancer stem cells in prostate cancer radioresistance. Cancer Lett. 2019;465:94-104.

19. Tahiliani M, Koh KP, Shen Y, Pastor WA, Bandukwala H, Brudno Y, et al. Conversion of 5-methylcytosine to 5-hydroxymethylcytosine in mammalian DNA by MLL partner TET1. Science. 2009;324:930-5.

20. Szwagierczak A, Bultmann S, Schmidt CS, Spada F, Leonhardt H. Sensitive enzymatic quantification of 5-hydroxymethylcytosine in genomic DNA. Nucleic Acids Res. 2010;38:e181.

21. Lorsbach RB, Moore J, Mathew S, Raimondi SC, Mukatira ST, Downing JR. TET1, a member of a novel protein family, is fused to MLL in acute myeloid leukemia containing the $\mathrm{t}(10 ; 11)(\mathrm{q} 22 ; \mathrm{q} 23)$. Leukemia. 2003;17:637-41.

22. Wang K-C, Kang C-H, Tsai C-Y, Chou N-H, Tu Y-T, Li G-C, et al. Ten-eleven translocation 1 dysfunction reduces 5-hydroxymethylcytosine expression levels in gastric cancer cells. Oncol Lett. 2017;15:278-84.

23. Yokoyama S, Higashi M, Tsutsumida H, Wakimoto J, Hamada T, Wiest E, et al. TET1-mediated DNA hypomethylation regulates the expression of MUC4 in lung cancer. Genes Cancer. 2017;8:517-27. https://doi.org/10. 18632/genesandcancer.139.

24. Müller T, Gessi M, Waha A, Isselstein LJ, Luxen D, Freihoff D, et al. Nuclear exclusion of TET1 is associated with loss of 5-hydroxymethylcytosine in IDH1 wild-type gliomas. Am J Pathol. 2012;181:675-83. https://doi.org/10.1016/j.ajpath.2012.04.017.

25. Yu S, Yin Y, Hong S, Cao S, Huang Y, Chen S, et al. TET1 is a tumor suppressor that inhibits papillary thyroid carcinoma cell migration and invasion. Int J Endocrinol. 2020;2020:1-9.

26. Murata A, Baba Y, Ishimoto T, Miyake K, Kosumi K, Harada K, et al. TET family proteins and 5-hydroxymethylcytosine in esophageal squamous cell carcinoma. Oncotarget. 2015;6:23372-82.

27. Good CR, Panjarian S, Kelly AD, Madzo J, Patel B, Jelinek J, Issa J-PJ. TET1-mediated hypomethylation activates oncogenic signaling in triple-negative breast cancer. Cancer Res. 2018;78:4126-37.

28. Hsu C-H, Peng K-L, Kang M-L, Chen Y-R, Yang Y-C, Tsai C-H, et al. TET1 suppresses cancer invasion by activating the tissue inhibitors of metalloproteinases. Cell Rep. 2012;2:568-79.
29. Spans L, van den BroeckT, Smeets E, Prekovic S, Thienpont B, Lambrechts $D$, et al. Genomic and epigenomic analysis of high-risk prostate cancer reveals changes in hydroxymethylation and TET1. Oncotarget. 2016;7:24326-38.

30. Lambert SA, Jolma A, Campitelli LF, Das PK, Yin Y, Albu M, et al. The human transcription factors. Cell. 2018;172:650-65.

31. Touzet $H$, Varré J-S. Efficient and accurate $p$ value computation for position weight matrices. Algorithms Mol Biol. 2007;2:1-12.

32. Diez D, Hutchins AP, Miranda-Saavedra D. Systematic identification of transcriptional regulatory modules from protein-protein interaction networks. Nucleic Acids Res. 2014;42:e6-e6.

33. Huang DW, Sherman BT, Lempicki RA. Bioinformatics enrichment tools: paths toward the comprehensive functional analysis of large gene lists. Nucleic Acids Res. 2009:37:1-13.

34. Huang DW, Sherman BT, Lempicki RA. Systematic and integrative analysis of large gene lists using DAVID bioinformatics resources. Nat Protoc. 2009;4:44-57.

35. Liberzon A, Birger C, Thorvaldsdóttir H, Ghandi M, Mesirov JP, Tamayo P. The molecular signatures database (MSigDB) hallmark gene set collection. Cell Syst. 2015;1:417-25.

36. Kanehisa M. KEGG: kyoto encyclopedia of genes and genomes. Nucleic Acids Res. 2000;28:27-30.

37. Oughtred R, Rust J, Chang C, Breitkreutz B-J, Stark C, Willems A, et al. The BioGRID database: a comprehensive biomedical resource of curated protein, genetic, and chemical interactions. Protein Sci. 2021;30:187-200.

38. Amoutzias GD, Robertson DL, van de Peer Y, Oliver SG. Choose your partners: dimerization in eukaryotic transcription factors. Trends Biochem Sci. 2008;33:220-9.

39. Feng J, Wang Q, Li G, Zeng X, Kuang S, Li X, Yue Y. TET1-mediated different transcriptional regulation in prostate cancer. Int J Clin Exp Med. 2015:8:203-11.

40. Dhiman VK, Attwood K, Campbell MJ, Smiraglia DJ. Hormone stimulation of androgen receptor mediates dynamic changes in DNA methylation patterns at regulatory elements. Oncotarget. 2015;6:42575-89.

41. Wang $K$, Chen Z, Shi J, Feng Y, Yu M, Sun Y, et al. Resveratrol inhibits the tumor migration and invasion by upregulating TET1 and reducing TIMP2/3 methylation in prostate carcinoma cells. Prostate. 2020;80:977-85.

42. Ross SE, Bogdanovic O. TET enzymes, DNA demethylation and pluripotency. Biochem Soc Trans. 2019:47:875-85.

43. Kaas GA, Zhong C, Eason DE, Ross DL, Vachhani RV, Ming G-L, et al. TET1 controls CNS 5-methylcytosine hydroxylation, active DNA demethylation, gene transcription, and memory formation. Neuron. 2013;79:1086-93.

44. Fu H-L, Ma Y, Lu L-G, Hou P, Li B-J, Jin W-L, Cui D-X. TET1 exerts its tumor suppressor function by interacting with $\mathrm{p} 53-\mathrm{EZH} 2$ pathway in gastric cancer. J Biomed Nanotechnol. 2014;10:1217-30.

45. Han X, Zhou Y, You Y, Lu J, Wang L, Hou H, et al. TET1 promotes cisplatinresistance via demethylating the vimentin promoter in ovarian cancer. Cell Biol Int. 2017:41:405-14.

46. Wu J, Li X, Huang H, Xia X, Zhang M, Fang X. TET1 may contribute to hypoxia-induced epithelial to mesenchymal transition of endometrial epithelial cells in endometriosis. PeerJ. 2020;8:e9950.

47. Arioka Y, Watanabe A, Saito K, Yamada Y. Activation-induced cytidine deaminase alters the subcellular localization of Tet family proteins. PLoS ONE. 2012;7:e45031.

48. Li H-J, Wang Y, Li B-X, Yang Y, Guan F, Pang X-C, Li X. Roles of ten-eleven translocation family proteins and their O-linked $\beta-N$ acetylglucosaminylated forms in cancer development. Oncol Lett. 2021;21:1.

49. Bao B, Teslow EA, Mitrea C, Boerner JL, Dyson G, Bollig-Fischer A. Role of TET1 and $5 \mathrm{hmC}$ in an obesity-linked pathway driving cancer stem cells in triple-negative breast cancer. Mol Cancer Res. 2020;18(12):1803-14.

50. Teslow EA, Mitrea C, Bao B, Mohammad RM, Polin LA, Dyson G, Purrington KS, Bollig-Fischer A. Obesity-induced MBD2_v2 expression promotes tumor-initiating triple-negative breast cancer stem cells. Mol Oncol. 2019;13(4):894-908.

51. Wang H, An X, Yu H, Zhang S, Tang B, Zhang X, Li Z. MiR-29b/TET1/ZEB2 signaling axis regulates metastatic properties and epithelial-mesenchymal transition in breast cancer cells. Oncotarget. 2017;8(60):102119-33.

52. To PK, Do MH, Cho J-H, Jung C. Growth modulatory role of zinc in prostate cancer and application to cancer therapeutics. IJMS. 2020;21:2991. 
53. Gardiner BJ, Herrick KW, Bailey RC, Chow JK, Snydman DR. Reactivation of latent cytomegalovirus infection after major surgery: risk factors and outcomes. Surg Infect. 2019;20:416-23.

54. Read SA, Obeid S, Ahlenstiel C, Ahlenstiel G. The role of zinc in antiviral immunity. Adv Nutr. 2019;10:696-710.

\section{Publisher's Note}

Springer Nature remains neutral with regard to jurisdictional claims in published maps and institutional affiliations.

- fast, convenient online submission

- thorough peer review by experienced researchers in your field

- rapid publication on acceptance

- support for research data, including large and complex data types

- gold Open Access which fosters wider collaboration and increased citations

- maximum visibility for your research: over 100M website views per year

At BMC, research is always in progress.

Learn more biomedcentral.com/submissions 